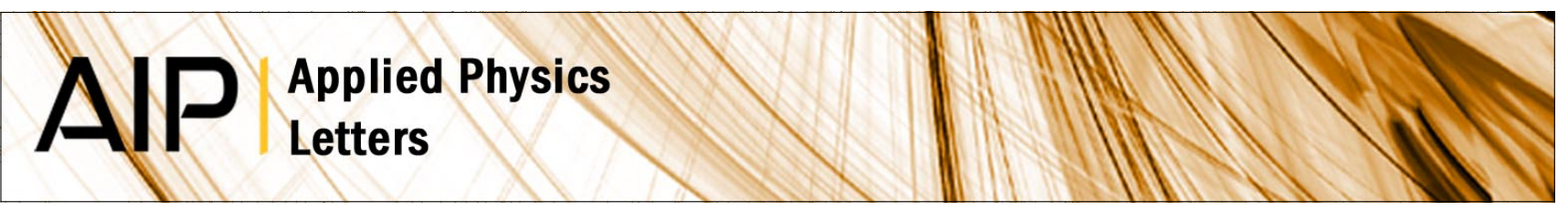

\title{
Electron channeling contrast imaging studies of nonpolar nitrides using a scanning electron microscope
}

G. Naresh-Kumar, C. Mauder, K. R. Wang, S. Kraeusel, J. Bruckbauer et al.

Citation: Appl. Phys. Lett. 102, 142103 (2013); doi: 10.1063/1.4801469

View online: http://dx.doi.org/10.1063/1.4801469

View Table of Contents: http://apl.aip.org/resource/1/APPLAB/v102/i14

Published by the American Institute of Physics.

Additional information on Appl. Phys. Lett.

Journal Homepage: http://apl.aip.org/

Journal Information: http://apl.aip.org/about/about_the_journal

Top downloads: http://apl.aip.org/features/most_downloaded

Information for Authors: http://apl.aip.org/authors

\section{ADVERTISEMENT}

\section{AIP Applied Physics Letters}

\section{EXPLORE WHAT'S NEW IN APL}

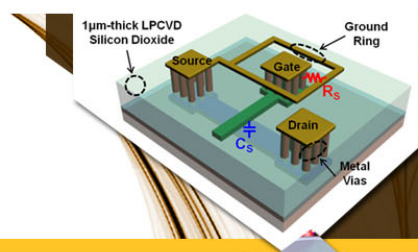

SURFACES AND INTERFACES

Focusing on physical, chemical, biological

structural, optical, magnetic and electrical

properties of surfaces and interfaces, and more.. 


\title{
Electron channeling contrast imaging studies of nonpolar nitrides using a scanning electron microscope
}

\author{
G. Naresh-Kumar, ${ }^{1, a)}$ C. Mauder ${ }^{2,3}$ K. R. Wang, ${ }^{4}$ S. Kraeusel, ${ }^{1}$ J. Bruckbauer, ${ }^{1}$ \\ P. R. Edwards, ${ }^{1}$ B. Hourahine, ${ }^{1}$ H. Kalisch, ${ }^{2}$ A. Vescan, ${ }^{2}$ C. Giesen, ${ }^{3}$ M. Heuken, ${ }^{2,3}$ \\ A. Trampert, ${ }^{4}$ A. P. Day, ${ }^{5}$ and C. Trager-Cowan ${ }^{1}$ \\ ${ }^{1}$ Department of Physics, SUPA, University of Strathclyde, Glasgow G4 ONG, United Kingdom \\ ${ }^{2}$ GaN Device Technology, RWTH Aachen University, Sommerfeldstr. 24, 52074 Aachen, Germany \\ ${ }^{3}$ AIXTRON SE, Kaiserstr. 98, 52134 Herzogenrath, Germany \\ ${ }^{4}$ Paul-Drude-Institut für Festkörperelektronik, Hausvogteiplatz 5-7, 10117 Berlin, Germany \\ ${ }^{5}$ Aunt Daisy Scientific Ltd., Claremont House, High St., Lydney, Gloucestershire GL15 5DX, United Kingdom
}

(Received 22 January 2013; accepted 26 March 2013; published online 9 April 2013)

\begin{abstract}
Threading dislocations, stacking faults, and associated partial dislocations significantly degrade the optical and electrical properties of materials such as non-polar III-nitride semiconductor thin films. Stacking faults are generally difficult to detect and quantify with existing characterization techniques. We demonstrate the use of electron channeling contrast imaging in the scanning electron microscope to non-destructively reveal basal plane stacking faults terminated by partial dislocations in m-plane $\mathrm{GaN}$ and $\mathrm{InGaN} / \mathrm{GaN}$ multiple quantum well structures grown on $\gamma-\mathrm{LiAlO}_{2}$ by metal organic vapor phase epitaxy. @ 2013 AIP Publishing LLC. [http://dx.doi.org/10.1063/1.4801469]
\end{abstract}

In the last two decades, III-nitride semiconductors have revolutionized the field of blue and green light emitters such as laser diodes (LDs) and light emitting diodes (LEDs). ${ }^{1-3} \mathrm{An}$ intrinsic problem with commercial nitride devices based on polar nitrides (c-plane orientation) is the presence of large internal electric fields which cause spatial separation of the electron and hole wavefunctions in the quantum well region. This is one of the phenomena currently limiting the efficiencies of nitride-based LDs and LEDs. In order to address this problem, growth of nonpolar nitrides (m-plane and a-plane) is being investigated. ${ }^{4,5}$ On top of performance issues, cost is an important factor for producing viable device structures. To date, m-plane III-nitride LEDs have been grown on substrates such as $\mathrm{Si}^{6}{ }^{6} \mathrm{~m}$-plane $\mathrm{SiC},{ }^{7}$ free standing m-plane $\mathrm{GaN}^{8}{ }^{8}$ a-plane sapphire, ${ }^{9}$ r-plane sapphire, ${ }^{10,11}$ and $\gamma-\mathrm{LiAlO}_{2} \cdot{ }^{4,12}$ Amongst these substrates, the use of Si and $\gamma-\mathrm{LiAlO}_{2}$ are considered to be the most economically viable. In addition to $\gamma-\mathrm{LiAlO}_{2}$ being inexpensive, growth on (100) $\mathrm{LiAlO}_{2}$ provides a substrate-lattice mismatch of only $1.7 \%$ and $0.3 \%$ in the [11-20] and [0001] directions of m-plane $\mathrm{GaN}$, respectively. ${ }^{12}$

Irrespective of the substrates or the growth plane employed, extended defects such as threading dislocations (TDs), stacking faults (SFs), and associated partial dislocations (PDs) are always present in the as-grown layers and have proven to be detrimental to device performance. ${ }^{13-16} \mathrm{In}$ nonpolar nitrides, $\mathrm{ZnO}$, and in $\mathrm{SiC}, \mathrm{SFs}$ and the associated PDs are of particular concern because of the higher densities of these defects in such materials. The capability to analyze TDs, PDs, and SFs and determine their densities nondestructively, rapidly, and without any sample preparation will remove a significant bottleneck to the development of high quality material. This will also help in understanding the fundamental structural properties related to microstructure evolution and thin film growth. To satisfy these requirements, we demonstrate the use of a scanning electron

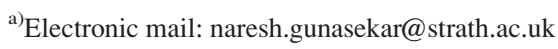

microscope (SEM) based diffraction technique known as electron channeling contrast imaging (ECCI) to characterize nonpolar (m-plane) $\mathrm{GaN}$ films and $\mathrm{InGaN} / \mathrm{GaN}$ multiple quantum well (MQW) structures.

Recent advancements have made X-ray diffraction (XRD) a powerful tool for characterizing nitride semiconductors, but there are several limitations in using XRD to characterize nonpolar nitrides. ${ }^{17}$ Modified Williamson-Hall analysis is a widely used method to estimate SF densities in nonpolar GaN thin films. ${ }^{18}$ However, the accuracy of this technique is limited to line densities above $10^{4} \mathrm{~cm}^{-1}$. Moreover, the applicability of this method is questionable as other superimposing effects, such as surface morphology and wafer bowing, may produce unphysical results. ${ }^{19}$

At present, transmission electron microscopy (TEM) is the most widely used technique for characterizing individual dislocations and SFs. ${ }^{20-23}$ High resolution TEM images are used to reveal the stacking sequence of the SFs and thus identify their types. ${ }^{24}$ However, difficulties in sample preparation and the localized nature of the information acquired from TEM make other microscopic imaging techniques worthwhile to explore.

ECCI is one of the emerging techniques for studying nitride semiconductors ${ }^{25-28}$ and other materials, such as $\mathrm{SiC}$ (Ref. 29) and $\mathrm{SrTiO}_{3}{ }^{30}$ In ECCI, images are produced from electrons which channel down the crystal planes of a suitably oriented sample. Changes in crystallographic orientation, or in lattice constant due to local strain, are revealed by changes in contrast in a channeling image constructed by monitoring the intensity of backscattered electrons as the electron beam is scanned over the sample. Extremely small changes in orientation and strain are detectable, revealing, for example, low-angle tilt, rotation boundaries, and atomic steps, and enabling dislocations and SFs to be imaged. ${ }^{28}$

The conditions required to resolve individual TDs and SFs using ECCI are quite stringent; a high brightness source is required to produce a small (nanometres), low divergence 
(of the order of few mrad) electron beam is required. ${ }^{25,28}$ Such conditions are met in a field emission gun (FEG) SEM. ECCI can be performed in either the backscatter geometry (sample at approximately $90^{\circ}$ to the impinging electron beam with the backscattered electrons detected by an electronsensitive diode or diodes placed under the pole piece of the microscope) or forescatter geometry (sample is tilted between $30^{\circ}$ and $70^{\circ}$ to the impinging electron beam and the forescattered electrons detected by a diode placed in front of the sample). ${ }^{28,31}$ The forescatter geometry provides better signal to noise when compared to the backscattered geometry. Detailed reviews on ECCI which discuss the relative advantages and disadvantages of the forescatter and the backscatter geometries are given by Wilkinson and Hirsch ${ }^{32}$ and Simpkin and Crimp. ${ }^{31}$ It is also necessary to use a detection system that allows discrimination between those electrons leaving the sample which carry channeling information and those which have been diffusely scattered by the sample.

In the present work, m-plane GaN epilayers and m-plane InGaN/GaN MQW structures with different In composition were studied using ECCI in a forescatter geometry. XRD and TEM were used to obtain complementary data. m-plane GaN buffer layers of $0.9 \mu \mathrm{m}$ were grown on top of (100) $\gamma-\mathrm{LiAlO}_{2}$ substrates using metal organic vapor phase epitaxy (MOVPE). The MQW structures consist of 5 pairs of 4-nm-thick InGaN wells and 16-nm-thick GaN barriers and were deposited on top of the m-plane GaN buffer at different temperatures to vary the In content. A detailed description of the growth procedure and optical properties of the samples can be found elsewhere. $^{33,34}$ All the electron channeling contrast images were acquired with an electron beam spot of $\approx 4 \mathrm{~nm}$, a beam current of $\approx 2.5 \mathrm{nA}$, and a beam divergence of $\approx 4 \mathrm{mrad}$ using an FEI Sirion FEG-SEM. The forescatter diodes and signal amplifier used for ECCI were provided by KE Developments Ltd. XRD measurements were performed with a PANalytical X'Pert MRD Pro in open detector geometry. Cross-sectional TEM studies were performed using a JEOL 3010 transmission electron microscope with samples prepared by standard mechanical polishing and Ar ion beam thinning at $3 \mathrm{keV}$.

In order to understand the diffraction contrast mechanisms for imaging SFs using ECCI, it is necessary to know their crystallographic nature. SFs are planar defects; in the case of $\mathrm{GaN}$ they can be considered as a hexagonal packed stacking sequence of Ga-N bilayers. There are two types of SFs, namely basal plane stacking faults (BSFs) and prismatic stacking faults (PSFs). BSFs can be further classified into intrinsic and extrinsic types, and the intrinsic BSFs can be subdivided into type $\mathrm{I}_{1}$ and $\mathrm{I}_{2}$. The majority of the observed BSFs in nonpolar GaN are $\mathrm{I}_{1}$ as these have the lowest formation energy. ${ }^{35}$ The structure of an $\mathrm{I}_{1}$-type BSF involves the removal of one basal plane, displacing the crystal lattice by $1 / 2$ [0001] and an additional slip along $1 / 3[1-100]$, which adds up to a displacement vector $\boldsymbol{R}$ of $1 / 6$ [2-203]. Figure 1(a) shows a schematic of a perfect wurtzite lattice and a BSF of type $I_{1}$. The...ABABABAB... stacking sequence for the perfect structure changes to... $\mathrm{ABABCBCBC} \ldots$ with the boundary plane being terminated on its two sides by a $\mathrm{PD}$ with $\boldsymbol{b}=1 / 6[2-203]$.

Figure 1(b) shows an electron channeling contrast image for the m-plane GaN buffer layer revealing a striated pattern
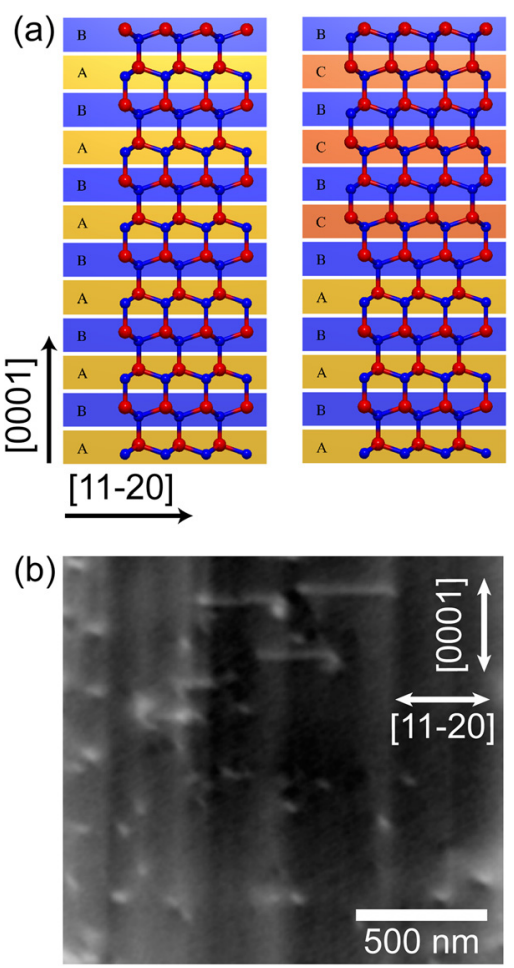

FIG. 1. (a) Schematic diagram of a perfect wurtzite lattice (left) and the faulted region (right) with an $\mathrm{I}_{1} \mathrm{BSF}$ introduced by changing the stacking sequence from $\mathrm{ABAB} \ldots$ to $\mathrm{BCBC} \ldots$ (b) ECCI of m-plane GaN layer exhibiting BSFs and TDs. The stripe direction was determined by XRD measurements.

along [0001] which is related to the anisotropic growth mode of m-plane $\mathrm{GaN}$ on $\mathrm{LiAlO}_{2}{ }^{12,36} \mathrm{BSF}$ in ECCI appear as lines with a PD terminating each end, and TDs appear as spots with black-white (B-W) contrast. A high magnification image of BSFs running along [11-20] (which is the basal plane direction for $\mathrm{m}$-plane $\mathrm{GaN}$ ), from an m-plane $\mathrm{In}_{0.04} \mathrm{Ga}_{0.96} \mathrm{~N} / \mathrm{GaN} \mathrm{MQW}$, is shown in Fig. 2(a). The contrast mechanism for BSFs is similar to TDs as both of them strain the crystal lattice. BSFs can either appear as a line with B-W contrast as shown in Fig. 1(b) or just as a black or white line as marked with dashed and dotted white circles in Fig. 2(a). Detailed descriptions of contrast mechanisms for TDs are given in Refs. 28, 32, 37, and 38. Dislocations and SFs are crystallographic features and generally do not appear in secondary electron (SE) images which are sensitive primarily to topography. Fig. 2(b) shows the corresponding SE image taken from the same region as the channeling contrast image shown in Fig. 2(a). While the stripes along [0001] (and along [11-20], discussed later) are discernible in the SE image due to their topography, on comparing the two images it becomes clear that BSFs only show observable contrast in the electron
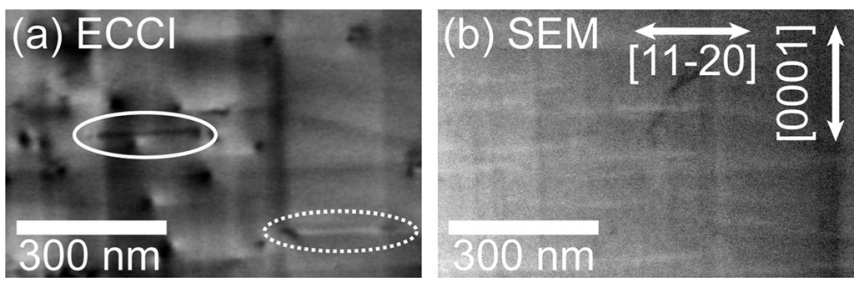

FIG. 2. (a) High magnification ECC image of $\operatorname{In}_{0.04} \mathrm{Ga}_{0.96} \mathrm{~N} / \mathrm{GaN}$ MQW and (b) SEM image of the same area. 
channeling contrast images. The contrast for TDs and BSFs is due to channeling; if the sample is tilted or rotated (moved away) from the channeling conditions, the TDs and BSFs disappear, confirming that these features are indeed imaged by channeled electrons. Note that it is also possible to observe channeling contrast in SE images, ${ }^{38}$ but in general, due to detection conditions, the contrast is low and difficult to discern.

In order to confirm the type of BSFs which are present in our samples, cross-sectional TEM was performed on an $\mathrm{m}$-plane $\mathrm{In}_{0.10} \mathrm{Ga}_{0.90} \mathrm{~N} / \mathrm{GaN}$ MQW sample. Figure 3 shows a high resolution cross-sectional TEM lattice image which shows the stacking sequence of crystal planes. The circles highlight the stacking sequence for an $\mathrm{I}_{1} \mathrm{SF}$. No other types of BSF were observed in this structure for the area analyzed using TEM. Moreover, the lateral extension of BSFs revealed by ECCI is always in the same range (of the order of $300 \mathrm{~nm}$ in length), so it can be assumed that all BSFs observed by ECCI are of the same type, namely $\mathrm{I}_{1}$.

Care has to be taken when counting TDs and SFs to build up statistically significant values for the TD and BSF densities. Series of electron channeling contrast images from random locations on the samples were acquired for this purpose. SF densities are typically represented as line densities $\left(\mathrm{cm}^{-1}\right)$ which are calculated by dividing the SF area by the probed volume of the sample. In TEM, BSF densities can be measured from plan view and/or cross-sectional images, whereas in ECCI we estimate the BSF densities purely from plan view images. As ECCI can yield information from a larger field of view, up to of order $500 \mu \mathrm{m}^{2}$, statistically significant numbers for defect densities can be estimated. In the present work, TD, BSF, and PD densities were estimated from several images each with an area of $\approx 5 \mu \mathrm{m}^{2}$, similar to the electron channeling contrast image shown in Fig. 1(b). The TD density for the m-plane GaN buffer layer was found to be $2.1 \pm 0.3 \times 10^{9} \mathrm{~cm}^{-2}$. Assuming the BSFs propagate through the entire sample, their line density was estimated to be $\approx 0.6 \times 10^{4} \mathrm{~cm}^{-1}$. The application of the XRD Williamson-Hall method, employing the (1-100) and (2-200) $\omega$-scan peak, provided an estimated BSF density of $\approx 1 \times 10^{4} \mathrm{~cm}^{-1}$. In order to increase the reliability

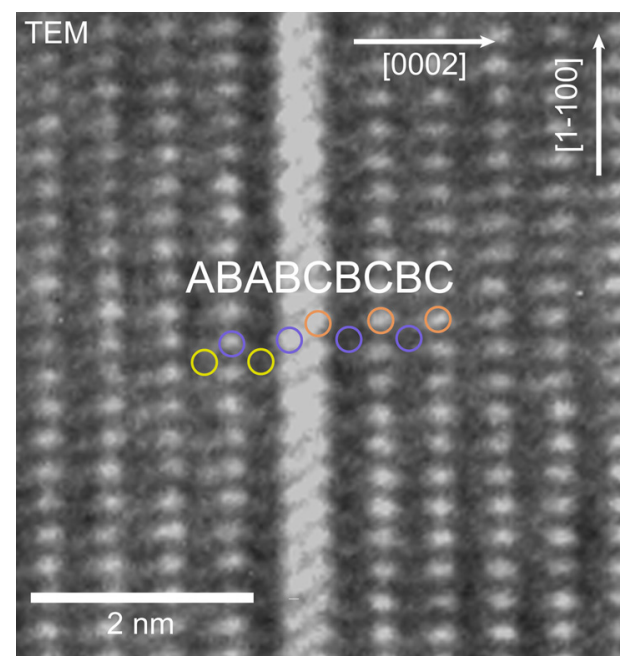

FIG. 3. High resolution cross-sectional TEM image showing the stacking mismatch. The atomic layers are indicated by similar colors as those used in Fig. 1(a). of our analysis without any assumptions, BSF number densities (area densities) ${ }^{39}$ were also estimated by simply counting the total number of BSFs appearing in the entire field of view in our ECCI images. For the m-plane GaN buffer layer, the BSF number density was found to be $9 \pm 2.5 \times 10^{7} \mathrm{~cm}^{-2}$ with a corresponding PD density of $1.8 \pm 0.5 \times 10^{8} \mathrm{~cm}^{-2}$; this is an order of magnitude lower than the TD density.

The TD density and the BSF number densities for an m-plane $\operatorname{In}_{0.04} \mathrm{Ga}_{0.96} \mathrm{~N} / \mathrm{GaN}$ MQW structure (the high magnification image shown in Fig. 2(a) is from this sample) were found to be $2.5 \pm 0.3 \times 10^{9} \mathrm{~cm}^{-2}$ and $10 \pm 4 \times 10^{7} \mathrm{~cm}^{-2}$, respectively, and thus in a very similar range to that of the $\mathrm{m}$-plane GaN buffer layer. The BSF line density was estimated to be $\approx 0.9 \times 10^{4} \mathrm{~cm}^{-1}$. For this structure, it was not possible to obtain an estimate of the BSF line density by XRD.

For the MQW structures, in addition to the striated features along [0001] which stem from the GaN buffer layer, another type of stripe (surface steps) is observed along [11-20] as shown in Fig. 2 (best observed in Fig. 4(a)). These features are only observed for the MQW samples, which indicate that they are either related to the lower growth temperature (required for the growth of $\mathrm{InGaN}_{\text {) }}$ or the presence of In during growth. An induced change in the anisotropic barrier height for adatom diffusion on the surface may be considered as an
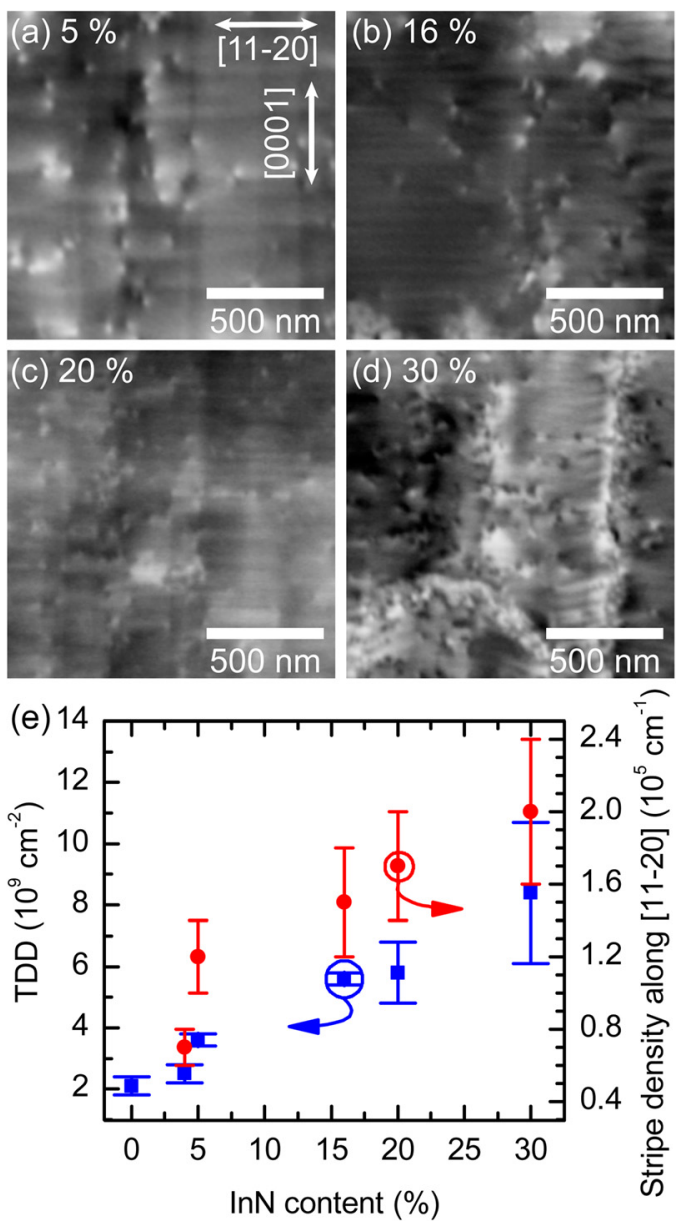

FIG. 4. (a)-(d) ECC images showing TDs and striated features along [0001] and [11-20]. (e) Graph showing the increase of TDs and stripe density along [11-20] with error bars as $\mathrm{InN} \%$ is increased. The values are calculated from channeling images. 
explanation for the observation of these features, but the exact origin is still under investigation. Although the direction of these features is identical to the BSF extension, the local positions of BSFs were found to be independent of the surface striations along [11-20] as revealed by the electron channeling contrast image shown in Fig. 2(a). It is, therefore, very unlikely that these striated features are related to the BSFs.

The density of the striated features along [11-20] and also the overall surface roughness increases with $\mathrm{InN}$ content. This makes it difficult to identify BSFs for samples with MQWs containing more than $5 \%$ InN. Although the surface roughness is of the order of $2 \mathrm{~nm}$ for a $1 \times 1 \mu \mathrm{m}^{2}$ area estimated from AFM images (not shown), it was still possible to estimate the TD densities by ECCI. Figures 4(a)-4(d) show electron channeling contrast images for InGaN/GaN MQWs with 5\%, 16\%, 20\%, and 30\% InN content, respectively. In Fig. 4(e), the average TD density values are plotted against the $\mathrm{InN}$ fraction, with the $0 \% \mathrm{InN}$ fraction representing the $\mathrm{GaN}$ buffer layer. As the $\mathrm{InN}$ fraction increases, higher numbers of TDs are observed which are of the order of $\approx 5-7 \times 10^{9} \mathrm{~cm}^{-2}$ for MQWs with $20 \%-30 \%$ of InN. The obvious increase in TD density for the MQW samples shows that additional defects are generated during the MQW growth. A reduced growth temperature and larger lattice strain may be responsible for the decrease in crystal quality. Since we were unable to identify BSFs in MQW samples with InN contents above about 5\%, we cannot rule out the possibility that these dislocations are caused by an increase in BSF density, which would also result in more PDs. Feng et al. recently performed cross-sectional TEM analysis of m-plane $\mathrm{In}_{0.26} \mathrm{Ga}_{0.74} \mathrm{~N} / \mathrm{GaN}$ MQWs and found that BSFs are formed within the MQWs. ${ }^{23}$

The stripe densities along [11-20] were also determined from both SEM and AFM images, which are of the order of $1-2 \times 10^{5} \mathrm{~cm}^{-1}$. This increase in the stripe densities along [11-20] is probably related to changes in the growth conditions required for higher $\mathrm{InN}$ incorporation in the MQWs.

In summary, we have demonstrated that ECCI is an ideal and statistically reliable technique for rapid and nondestructive quantification of TD and SF densities in nonpolar nitrides when compared to the presently available techniques. TEM measurements show that for our samples, the observed BSFs are predominantly of $\mathrm{I}_{1}$-type. The production of reasonable quality MOVPE InGaN/GaN MQW structures (InN content $\approx 20 \%$ ) grown on $\gamma-\mathrm{LiAlO}_{2}$ with TD densities in the order of $\approx 5 \times 10^{9} \mathrm{~cm}^{-2}$ is shown to be possible. We believe that the ECCI technique will be of great benefit for the development of high quality semiconductor materials and is particularly useful for the detection and quantification-and thereby eventual reduction — of stacking faults in nonpolar materials. ECCI may also be used in combination with cathodoluminescence and electric beam induced current to understand the evolution of extended defects and its role in determining the optical and electronic properties of semiconductor materials.

This work was carried out with the partial support of the EU under the ITN RAINBOW (http://rainbow.ensicaen.fr/), Grant Agreement No. PITN-GA-2008-213238.
${ }^{1}$ S. Nakamura, M. Senoh, S. Nagahama, N. Iwasa, T. Yamada, T. Matsushita, H. Kityoku, and Y. Sugimoto, Jpn. J. Appl. Phys., Part 2 35, L74 (1996).

${ }^{2}$ F. A. Ponce and D. P. Bour, Nature 386, 351 (1997).

${ }^{3}$ A. Khan, K. Balakrishnan, and T. Katona, Nat. Photonics 2, 77 (2008).

${ }^{4}$ P. Waltereit, O. Brandt, A. Trampert, H. T. Grahn, J. Menniger, M. Ramsteiner, M. Reiche, and K. H. Ploog, Nature 406, 865 (2000).

${ }^{5}$ J. S. Speck and S. F. Chichibu, MRS Bull. 34, 304 (2009).

${ }^{6}$ X. Ni, X. Ni, J. Lee, M. Wu, X. Li, R. Shimada, Ü. Özgür, A. A. Baski, H. Morkoc, T. Paskova, G. Mulholland, and K. R. Evans, Appl. Phys. Lett. 95, 101106 (2009).

${ }^{7}$ M. McLaurin, T. E. Mates, and J. S. Speck, Appl. Phys. Lett. 86, 262104 (2005).

${ }^{8}$ M. McLaurin and J. S. Speck, Phys. Status Solidi (RRL) 1, 110 (2007).

${ }^{9}$ Y. Saito, K. Okuno, S. Boyama, N. Nakada, S. Nitta, Y. Ushida, and N. Shibata, Appl. Phys. Express 2, 041001 (2009).

${ }^{10}$ A. Chitnis, C. Chen, V. Adivarahan, M. Shatalov, E. Kuokstis, V. Mandavilli, J. Yang, and M. A. Khan, Appl. Phys. Lett. 84, 3663 (2004).

${ }^{11}$ A. Chakraborty, B. A. Haskell, S. Keller, J. S. Speck, S. P. DenBaars, S. Nakamura, and U. K. Mishra, Appl. Phys. Lett. 85, 5143 (2004).

${ }^{12}$ Y. J. Sun, O. Brandt, U. Jahn, T. Y. Liu, A. Trampert, S. Cronenberg, S. Dhar, and K. H. Ploog, J. Appl. Phys. 92, 5714 (2002).

${ }^{13}$ S. Nakamura, Science 281, 956 (1998).

${ }^{14}$ D. C. Look and J. R. Sizelove, Phys. Rev. Lett. 82, 1237 (1999).

${ }^{15}$ K. H. Baik, Y. G. Seo, S. Hong, S. Lee, J. Kim, J. Son, and S. Hwang, IEEE Photon. Technol. Lett. 22, 595 (2010).

${ }^{16}$ J. Kioseoglou, E. Kalesaki, L. Lymperakis, J. Neugebauer, Ph. Komninou, and Th. Karakostas, J. Appl. Phys. 109, 083511 (2011).

${ }^{17}$ V. M. Kaganer, O. Brandt, A. Trampert, and K. H. Ploog, Phys. Rev. B 72, 045423 (2005).

${ }^{18}$ M. B. McLaurin, A. Hirai, E. Young, F. Wu, and J. Speck, Jpn. J. Appl. Phys., Part 1 47, 5429 (2008).

${ }^{19}$ M. A. Moram, C. F. Johnston, J. L. Hollander, M. J. Kappers, and C. J. Humphreys, J. Appl. Phys. 105, 113501 (2009).

${ }^{20}$ F. A. Ponce, D. Cherns, W. T. Young, and J. W. Steeds, Appl. Phys. Lett. 69, 770 (1996).

${ }^{21}$ D. M. Follstaedt, N. A. Missert, D. D. Koleske, C. C. Mitchell, and K. C. Cross, Appl. Phys. Lett. 83, 4797 (2003).

${ }^{22}$ R. Datta, M. J. Kappers, J. S. Barnard, and C. J. Humphreys, Appl. Phys. Lett. 85, 3411 (2004).

${ }^{23}$ F. Wu, Y. D. Lin, A. Chakraborty, H. Ohta, S. P. DenBaars, S. Nakamura, and J. S. Speck, Appl. Phys. Lett. 96, 231912 (2010).

${ }^{24}$ K. Suzuki, M. Ichihara and S. Takeuchi, Jpn. J. Appl. Phys., Part 1 33, 1114 (1994).

${ }^{25}$ C. Trager-Cowan, F. Sweeney, P. W. Trimby, A. P. Day, A. Gholinia, N.-H. Schmidt, P. J. Parbrook, A. J. Wilkinson, and I. M. Watson, Phys. Rev. B 75, 085301 (2007).

${ }^{26}$ Y. N. Picard, J. D. Caldwell, M. E. Twigg, C. R. Eddy, Jr., M. A. Mastro, R. L. Henry, and R. T. Holm, Appl. Phys. Lett. 91, 094106 (2007).

${ }^{27}$ G. Naresh-Kumar, B. Hourahine, A. Vilalta-Clemente, P. Ruterana, P. Gamarra, C. Lacam, M. Tordjman, M. A. di Forte-Poisson, P. J. Parbrook, A. P. Day, G. England, and C. Trager-Cowan, Phys. Status Solidi A 209, 424 (2012).

${ }^{28}$ G. Naresh-Kumar, B. Hourahine, P. R. Edwards, A. P. Day, A. Winkelmann, A. J. Wilkinson, P. J. Parbrook, G. England, and C. Trager-Cowan, Phys. Rev. Lett. 108, 135503 (2012).

${ }^{29}$ Y. N. Picard and M. E. Twigg, J. Appl. Phys. 104, 124906 (2008).

${ }^{30}$ R. J. Kamaladasa, W. Jiang, and Y. Picard, J. Electron. Mater. 40, 2222 (2011).

${ }^{31}$ B. A. Simpkin and M. A. Crimp, Ultramicroscopy 77, 65 (1999).

${ }^{32}$ A. J. Wilkinson and P. B. Hirsch, Micron 28, 279 (1997).

${ }^{33}$ C. Mauder, B. Reuters, K. R. Wang, D. Fahle, A. Trampert, M. V. Rzheutskii, E. V. Lutsenko, G. P. Yablonskii, J. F. Woitok, M. M. C. Chou, M. Heuken, H. Kalisch, and R. H. Jansen, J. Cryst. Growth 315, 246 (2011).

${ }^{34}$ C. Mauder, B. Reuters, L. R. Khoshroo, M. V. Rzheutskii, E. V. Lutsenko, G. P. Yablonskii, J. F. Woitok, M. Heuken, H. Kalisch, and R. H. Jansen, J. Cryst. Growth 312, 1823 (2010).

${ }^{35}$ C. Stampfl and C. G. Van de Walle, Phys. Rev. B 57, R15052 (1998).

${ }^{36}$ K. R. Wang, M. Ramsteiner, C. Mauder, Q. Wan, T. Hentschel, H. T. Grahn, H. Kalisch, M. Heuken, R. H. Jansen, and A. Trampert, Appl. Phys. Lett. 96, 231914 (2010).

${ }^{37}$ E. Ruedl, P. Delavignette, and S. Amelinckx, J. Nucl. Mater. 6, 46 (1962).

${ }^{38}$ D. C. Joy, D. E. Newbury, and D. L. Davidson, J. Appl. Phys. 53, R81 (1982).

${ }^{39}$ L. H. Kuo, K. Kimura, A. Ohtake, S. Miwa, T. Yasuda, and T. Yao, J. Vac. Sci. Technol. B 15, 1241 (1997). 